\title{
Combining functional features of whole-grain barley and legumes for dietary reduction of cardiometabolic risk: a randomised cross-over intervention in mature women
}

\author{
Juscelino Tovar ${ }^{1 *}$, Anne Nilsson ${ }^{2}$, Maria Johansson ${ }^{1}$ and Inger Björck ${ }^{1,2}$ \\ ${ }^{1}$ Antidiabetic Food Centre, Lund University, PO Box 124, SE-221 OO Lund, Sweden \\ ${ }^{2}$ Department of Applied Nutrition and Food Chemistry, Lund University, SE-221 OO Lund, Sweden \\ (Submitted 13 February 2013 - Final revision received 8 July 2013 - Accepted 21 August 2013 - First published online 24 September 2013)
}

\begin{abstract}
The usefulness of dietary strategies against cardiometabolic risk is increasingly being acknowledged. Legumes and whole grains can modulate risk markers associated with cardiometabolic diseases, but their possible additive/synergistic actions are unknown. The objective of the present study was to assess, in healthy subjects, the effect of a diet including specific whole-grain barley products and legumes with prior favourable outcomes on cardiometabolic risk parameters in semi-acute studies. A total of forty-six overweight women (50-72 years, BMI $25-33 \mathrm{~kg} / \mathrm{m}^{2}$ and normal fasting glycaemia) participated in a randomised cross-over intervention comparing a diet rich in kernel-based barley products, brown beans and chickpeas (D1, diet 1 (functional diet)) with a control diet (D2, diet 2 (control diet)) of similar macronutrient composition but lacking legumes and barley. D1 included $86 \mathrm{~g}$ (as eaten)/d brown beans, $82 \mathrm{~g} / \mathrm{d}$ chickpeas, $58 \mathrm{~g} / \mathrm{d}$ whole-grain barley kernels and $216 \mathrm{~g} / \mathrm{d}$ barley kernel bread. Both diets followed the Nordic Nutrition Recommendations, providing similar amounts of dietary fibre (D1: $46.9 \mathrm{~g} / \mathrm{d} ; \mathrm{D} 2: 43.5 \mathrm{~g} / \mathrm{d}$ ), with wheat-based products as the main fibre supplier in D2. Each diet was consumed for 4 weeks under weight-maintenance conditions. Both diets decreased serum total cholesterol, LDL-cholesterol and HDL-cholesterol levels, but D1 had a greater effect on total cholesterol and LDL-cholesterol levels $(P<0.001$ and $P<0 \cdot 05$, respectively). D1 also reduced apoB $(P<0 \cdot 001)$ and $\gamma$-glutamyl transferase $(P<0.05)$ levels, diastolic blood pressure $(P<0.05)$ and the Framingham cardiovascular risk estimate $(P<0.05)$. D1 increased colonic fermentative activity, as judged from the higher $(P<0 \cdot 001)$ breath hydrogen levels recorded. In conclusion, a specific barley/legume diet improves cardiometabolic risk-associated biomarkers in a healthy cohort, showing potential preventive value beyond that of a nutritionally well-designed regimen.
\end{abstract}

Key words: Cardiovascular risk: Dietary prevention: Legumes: Barley

Cardiometabolic diseases are important causes of adult disability and death in many countries. Among strategies for combating these complications, the dietary modulation of cardiometabolic risk has gained in popularity over the years $^{(1,2)}$. In addition to customary generic dietary regimens, such as the so-called Mediterranean and Nordic diets ${ }^{(3,4)}$, the potential of ad hoc-designed diets for the treatment and prevention of cardiometabolic disorders has attracted attention recently. For instance, a portfolio of plant foods has proven effective in reducing plasma cholesterol levels and cardiovascular risk in hypercholesterolaemics ${ }^{(5)}$. A diet combining different anti-inflammatory functional components has also been shown to have notable ability to ameliorate multiple metabolic risk parameters in healthy mature volunteers ${ }^{(6)}$.

An important aspect of risk management by dietary means is the degree of adherence to the prescribed regimen ${ }^{(2,7)}$. Therefore, documentation of the health-beneficial effects of common palatable foods is necessary, as good overall palatability and variety are key determinants of successful dietary compliance. Whole-grain cereals and legumes are interesting in this context, as they have been reported to be generally associated with health-beneficial effects ${ }^{(8-11)}$. Previous work carried out by this research group has investigated acute and semi-acute metabolic effects of different legumes and whole-grain cereals. It has been shown that a portion of intact boiled barley kernels eaten at dinner improves postprandial blood glucose control the following morning ${ }^{(12,13)}$ and promotes a significant reduction in the levels of circulating markers of inflammation ${ }^{(14)}$, features that may be useful in relation to the dietary management of metabolic risk. These effects seem to be related both to the low-glycaemic index properties of the cereal and to the colonic fermentation of its prebiotic carbohydrates. Legume seeds constitute another

Abbreviations: BP, blood pressure; D1, diet 1 (functional diet); D2, diet 2 (control diet); GGT, $\gamma$-glutamyl transferase.

* Corresponding author: J. Tovar, email juscelino.tovar@ffsc.lu.se 
promising ingredient, characterised by low glycaemic indices and significant fermentable substrate contents ${ }^{(9,15)}$. Recent data indicate that brown beans, in particular, exert noteworthy beneficial effects on postprandial plasma levels of biomarkers of inflammation, a phenomenon that may be associated with the perceived prebiotic features of the indigestible fraction of pulses $^{(16)}$. Similar effects have been recorded with chickpeas (A Nilsson, E Johansson, L Ekström and I Björck, unpublished results).

Combinatory approaches exploiting possible synergistic effects of conventional functional food items appear valuable in the design of successful regimens for reducing metabolic risk $^{(6)}$. Based on the aforementioned evidence, the present study examined the effects of a diet enriched with whole-grain kernel-based barley, brown beans and chickpeas on cardiometabolic risk-associated parameters in a healthy cohort of mature overweight women. The intervention followed a randomised cross-over design, pursuing the amelioration of cardiometabolic risk through improved dietary quality under body weight-maintenance conditions.

\section{Subjects and methods}

\section{Participants}

Non-smoking female volunteers without any known medical condition were recruited through advertisement in local newspapers and were informed orally and in writing of the project disposal, emphasising the non-weight-loss character of the investigation. Inclusion criteria were age between 50 and 73 years, BMI in the $25-33 \mathrm{~kg} / \mathrm{m}^{2}$ range and fasting plasma glucose values $\leq 6.1 \mathrm{mmol} / \mathrm{l}$. The only medications accepted were hormone replacement for thyroid problems (if constant during the whole trial) and prescription-free painkillers without anti-inflammatory action. The trial was initiated with fifty subjects. During the first week, two participants withdrew from the study: one disliked the barley/ legume dishes and the other faced personal problems. Another two subjects withdrew within a few days of starting the second phase of the trial. A total of forty-six completed the two phases of the trial and were included in the analysis (Fig. 1). Data obtained from non-completers were excluded. Baseline data collected at the time of the first clinical visit are given in Table 1.

\section{Study protocol}

The study was designed as a randomised, controlled, cross-over trial of the effect of a legume-whole-grain barley diet (D1, diet 1 (functional diet)) on markers related to cardiometabolic risk. The participants were randomly assigned, using a computerised random number generator, to one of two treatment orders starting with D1 or D2 (diet 2 (control diet)), respectively (Fig. 1). The randomisation was carried out by the study coordinator. Each dietary phase lasted 4 weeks with a 4-6-week wash-out period. The participants paid four visits to the clinical unit (Lund University Antidiabetic Food Centre), one before and one after each intervention period.
During each clinical visit, fasting body weight was recorded, and blood pressure (BP) was measured twice, while seated, in the non-dominant arm using a digital automatic BP monitor (Model M3 Intelligence, Omron Healthcare Company, Limited). A finger-tip capillary blood sample was collected for blood glucose measurement, and venous blood was then drawn for the assessment of fasting insulin, glycated $\mathrm{Hb}$, cholesterol (total, LDL and HDL), TAG, C-reactive protein, apoA1, apoB and $\boldsymbol{\gamma}$-glutamyl transferase (GGT) levels.

The consumption of dietary supplements such as fish oil (seven subjects), probiotic-containing products (twenty subjects) and herbal extracts (three subjects) was stopped 2 weeks before the start of the trial and was not resumed during the wash-out period. Before each dietary phase, the participants attended an introductory session with the nutritionist, who explained the practical details of the upcoming dietary period.

The participants were asked not to alter their physical activity habits during the study. A pretrial questionnaire indicated that $11 \%$ of the subjects had physical activity levels equivalent to $1 \mathrm{~h} / \mathrm{d}$ or more and $54 \%$ had levels equivalent to $30-60 \mathrm{~min} / \mathrm{d}$ and $35 \%$ reported a low activity level $(<30 \mathrm{~min} / \mathrm{d})$. The subjects were also instructed to check their fasting body weight weekly. Variations greater than $1 \mathrm{~kg}$ were reported to the nutritionist, who suggested compensatory dietary modifications. The intake of key components in both D1 (legumes and barley products) and D2 (wheat products) was not affected by these modifications, which were only necessary for five of the subjects.

The food items provided for the 4-week D1 dietary period were as follows: canned brown beans (Phaseolus vulgaris cv. Katja, Kalmar-Ölands Trädgårdsprodukter) and chickpeas (Cicer arietinum, Zeta-Di Lucca AB); whole barley seeds (Finax AB); an experimental whole-grain barley bread, baked by Credin A/S. Some of the foods included in D2 were as follows: wheat bread (18\% whole grain, Kneipp type; Pågen $\mathrm{AB}$ ); crisp rolls (60\% whole-grain wheat; Pågen AB); All-Bran Plus (Nordic Kellogg's). Additionally, coldpressed rapeseed oil (Dr PersFood AB) and fish-based spreads (Kalles Kaviar and Creamed Salmon; Abba Seafood AB) were provided during both the dietary periods.

To assess dietary compliance, the participants completed a daily menu checklist covering each 4-week dietary period. These checklists included the 'active components' of D1 and the dietary fibre-providing items in D2. They also completed a questionnaire investigating their experience with each diet, including an estimation of the satiating power of the diet when compared with that of their regular regimen ( -1 for 'less satiating than the habitual diet', 0 for 'equally satiating' and 1 for 'greater satiating power') ${ }^{(6)}$. Coaching support was provided by the nutritionist, who contacted each participant at least once per dietary period.

The present study was conducted according to the guidelines laid down in the Declaration of Helsinki, and all procedures involving human subjects were approved by the Regional Ethical Review Board, Lund, Sweden (Dnr 2011/140). Written informed consent was obtained from all the subjects. Recruitment began in April 2011, and the experimental trials took place between September 2011 and May 2012. The study was registered at ClinicalTrials.gov as NCT01527253. 


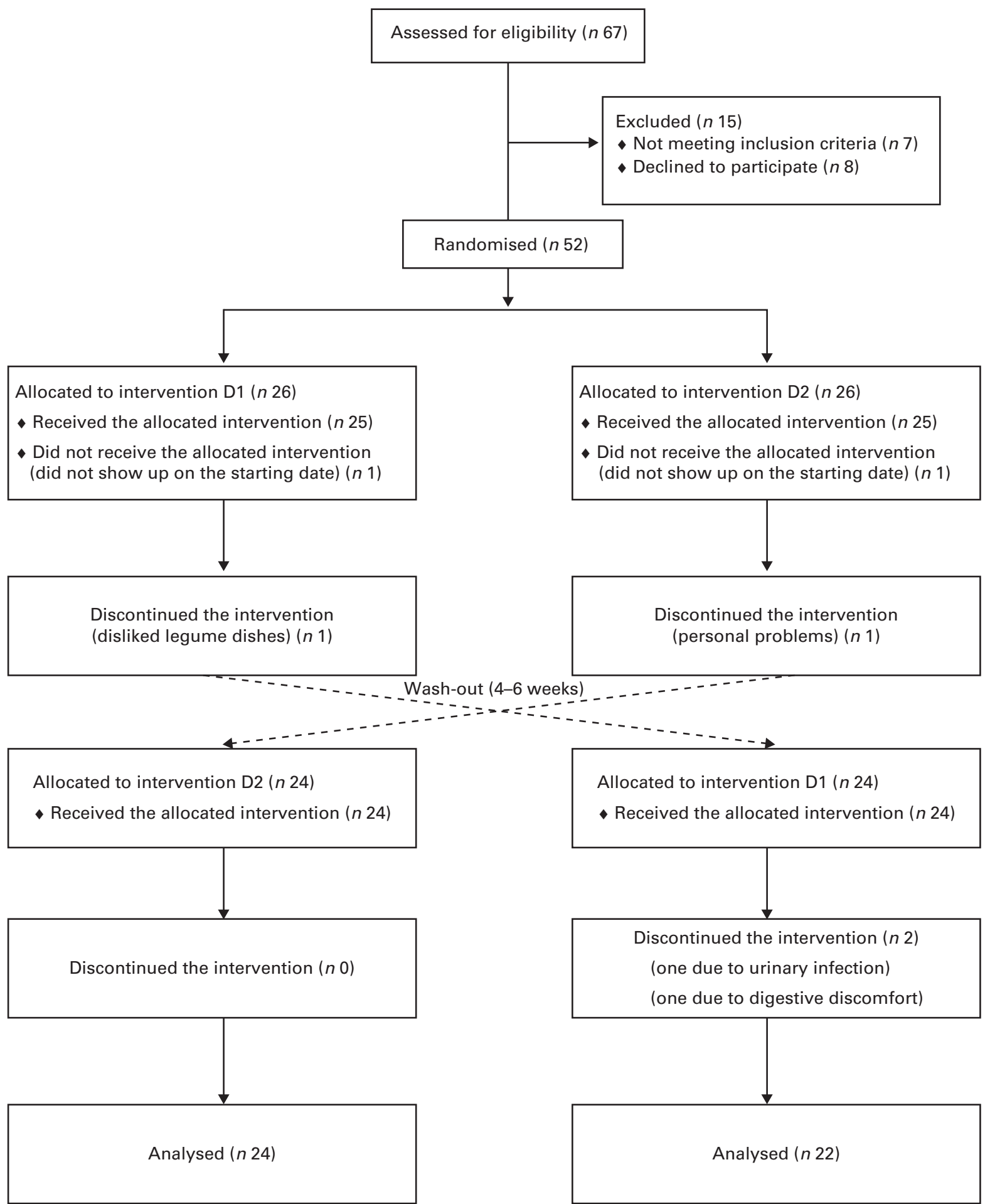

Fig. 1. Flow diagram of enrolment, random assignment, withdrawals and follow-up of the study participants. D1, diet 1 (functional diet); D2, diet 2 (control diet).

\section{Diets}

The participants ate their habitual diet before and between the experimental periods. A dietary habit questionnaire completed before randomisation to the starting dietary treatment was used to help the participants resume their eating habits during the wash-out period. To ensure good compliance, the participants were provided with a detailed 2 -week rotating menu plan for each dietary period (D1 and D2) with all the food ingredients expressed in weight and/or volume measures. Electronic self-rating scales were made available whenever needed. The menu plan also included recipes for preparing meals.

Both diets were designed in close agreement with the Nordic Nutrition Recommendations ${ }^{(17)}$ and supplied $8786 \mathrm{~kJ} /$ d $(2100 \mathrm{kcal} / \mathrm{d})$, combining foods from plant and animal 
Table 1. Characteristics of the participants at baseline

(Mean values with their standard errors, $n 46$ )

\begin{tabular}{lcc}
\hline & Mean & SEM \\
\hline Age (years) & 61.6 & 0.8 \\
Body weight (kg) & 78.3 & 1.6 \\
BMI (kg/m ${ }^{2}$ ) & 28.8 & 1.2 \\
Cholesterol (mmol/l) & & \\
$\quad$ Total & 6.39 & 0.25 \\
LDL & 3.99 & 0.24 \\
HDL & 1.84 & 0.03 \\
TAG (mmol/l) & 1.11 & 0.19 \\
Apo (g/l) & & \\
ApoA1 & 1.75 & 0.03 \\
ApoB & 1.10 & 0.09 \\
Insulin (mU/l) & 8.16 & 0.83 \\
Glucose (mmol/l) & 5.39 & 0.04 \\
HOMA-IR & 1.71 & 0.20 \\
HbA1c (\%) & 3.58 & 0.05 \\
CRP (mg/l) & 1.84 & 0.42 \\
GGT ( $\mu$ kat/l) & 0.33 & 0.01 \\
Blood pressure (mmHg) & & \\
$\quad$ Systolic & 137.1 & 1.9 \\
Diastolic & 83.3 & 1.6 \\
\hline HOMA-IR, homeostatic model assessment of insulin \\
$\quad$ resistance; HbA1c, glycated Hb; CRP, C-reactive \\
protein; GGT, $\gamma$-glutamyl transferase. \\
*1 mU/l = 6.945 pmol/l. \\
$\quad$
\end{tabular}

origins commonly available in food stores. D1 also included a whole-barley bread prototype. The nutritional profiles of D1 and D2 are given in Table 2.

On a daily basis, D1 included $86 \mathrm{~g}$ (as eaten) brown beans, $82 \mathrm{~g}$ chickpeas, $58 \mathrm{~g}$ whole-grain barley kernels and $216 \mathrm{~g}$ whole-grain barley kernel bread. This bread contained whole barley kernels and whole-grain barley flour blended with a minor proportion of wheat flour (15\% of the DM). Based on previous studies showing overnight beneficial effects on glycaemic regulation ${ }^{(12)}$ and circulating markers of inflammation ${ }^{(14,16)}$, the aforementioned daily supply of legumes and barley was selected to provide $100 \mathrm{~g}$ of available carbohydrates, i.e. $77 \mathrm{~g}$ from barley and $23 \mathrm{~g}$ from the legumes (Table 3). Both diets provided similar amounts of cholesterol (D1: $233 \mathrm{mg} / \mathrm{d}$; D2: $236 \mathrm{mg} / \mathrm{d}$; Table 2) and total dietary fibre (D1: $47 \mathrm{~g} / \mathrm{d}$; D2: $44 \mathrm{~g} / \mathrm{d}$ ) (Table 3), with wheat-based products as the main fibre supplier in D2.

The participants followed a $14 \mathrm{~d}$ rotating menu plan. A representative $1 \mathrm{~d}$ menu for $\mathrm{D} 1$ and $\mathrm{D} 2$ is given in Table 4. The participants were allowed to consume a limited amount of alcoholic beverages ( $30 \mathrm{~g}$ ethanol/week) during both the dietary periods. This limit, however, did not compel low drinkers to increase their usual ethanol consumption. Due to the low overall energy contribution from such contingent alcoholic drinks, they were not included in the estimation of the energy content of diets. The participants did not change their salt and coffee- and tea-drinking habits during the trial.

On the last day of the D1 dietary period, the participants ate an evening meal ( $1318 \mathrm{~kJ}$ ( $315 \mathrm{kcal}), 10 \mathrm{~g}$ dietary fibre) containing $86 \mathrm{~g}$ of the experimental barley bread, $28 \mathrm{~g}$ chickpeas and $29 \mathrm{~g}$ brown beans, accompanied by margarine and vinegar.
The D2 dietary period concluded with a dinner that included $34 \mathrm{~g}$ whole-grain wheat bread, $26 \mathrm{~g}$ whole-grain crisp rolls and $25 \mathrm{~g}$ All-Bran Plus $(1297 \mathrm{~kJ}$ (310 kcal), $11 \mathrm{~g}$ dietary fibre).

\section{Analyses}

Routine blood tests were carried out at the Clinical Chemistry Laboratory/Skåne University Hospital Lund on fasting plasma (total cholesterol and HDL-cholesterol, TAG, apoA1, apoB, C-reactive protein and GGT), serum (insulin) or total blood (glycated $\mathrm{Hb}$ ) samples. The concentrations of LDL-cholesterol were calculated $^{(18)}$. Homeostatic model assessment of insulin resistance was calculated from fasting blood plasma glucose and serum insulin values ${ }^{(19)}$.

The concentrations of fasting capillary plasma glucose were measured immediately after bleeding (HemoCue ${ }^{\circledR}$ B-glucose, HemoCue AB). Breath hydrogen levels were measured as an indicator of colonic fermentation, using an EC 60 Gastrolzser (Bedfont Scientific Ltd).

The compositions of the diets were analysed using the 2009 food database from the Swedish National Food Administration and a computerised calculation program (Dietist XP 3·1; Kost och Näringsdata $\mathrm{AB}$ ).

The contents of soluble and insoluble dietary fibres ${ }^{(20)}$, as well as available $\operatorname{starch}^{(21)}$, were determined in chickpeas, brown beans and barley products included in D1.

\section{Calculations and statistical analysis}

Results are expressed as means with their standard errors. Data were evaluated by a mixed-model ANOVA with sequence and interaction between diets and start/end of treatment periods as fixed effects and participants within sequence and visit as random effects. Least-squares means were estimated for the start (D1/week 0 and D2/week 0) and end

Table 2. Nutritional profiles of diet 1 (D1, functional diet) and diet 2 (D2, control diet)*

(Mean values with their standard errors)

\begin{tabular}{|c|c|c|c|c|}
\hline & \multicolumn{2}{|c|}{ D1 } & \multicolumn{2}{|c|}{$\mathrm{D} 2$} \\
\hline & Mean & SEM & Mean & SEM \\
\hline Energy $(\mathrm{kcal} / \mathrm{d}) \dagger$ & 2130 & 57 & 2140 & 44 \\
\hline Energy $(\mathrm{kJ} / \mathrm{d}) \dagger$ & 8912 & 238 & 8954 & 184 \\
\hline Protein (E\%) & 16 & 0.6 & 16 & 0.4 \\
\hline Carbohydrate (E\%) & 53 & $1 \cdot 3$ & 53 & 0.9 \\
\hline Fat $(E \%)$ & 31 & $1 \cdot 1$ & 31 & 0.9 \\
\hline Saturated fat (E\%) & 10 & 0.4 & 9 & 0.5 \\
\hline Monounsaturated fat (E\%) & 12 & 0.5 & 12 & 0.4 \\
\hline Polyunsaturated fat (E\%) & 7 & 0.3 & 6 & 0.3 \\
\hline$n-6$ Fatty acids (E\%) & 4.5 & 0.2 & $4 \cdot 1$ & 0.1 \\
\hline$n$-3 Fatty acids (E\%) & $2 \cdot 2$ & 0.2 & $2 \cdot 1$ & 0.1 \\
\hline$n-6: n-3$ ratio & $2 \cdot 1$ & 0.1 & $2 \cdot 1$ & 0.1 \\
\hline Dietary fibre (g/d) & 47 & 1.4 & 44 & 1.4 \\
\hline Cholesterol (mg/d) & 233 & 33 & 236 & 31 \\
\hline
\end{tabular}

$\mathrm{E} \%$, percentage of energy.

* Mean daily values from $14 \mathrm{~d}$ (2-week rotating menu plan) with their standard errors.

† Increased energy intake was prescribed for subjects who experienced weight loss during the initial weeks (see Study protocol section). 
Table 3. Starch and dietary fibre supplied by barley and legumes in diet 1 (functional diet) $(\mathrm{g} / \mathrm{d})$

\begin{tabular}{lccccc}
\hline & & \multicolumn{3}{c}{ Dietary fibre } \\
\cline { 3 - 5 } & $\begin{array}{c}\text { Available } \\
\text { starch }\end{array}$ & Soluble & Insoluble & Total \\
\hline Barley bread $(216 \mathrm{~g} / \mathrm{d})$ & 60 & 5 & 11 & 16 \\
Barley kernels $(58 \mathrm{~g} / \mathrm{d})$ & 17 & 1 & 2 & 3 \\
Brown beans $(86 \mathrm{~g} / \mathrm{d})$ & 12 & 2 & 5 & 7 \\
Chickpeas $(82 \mathrm{~g} / \mathrm{d})$ & 11 & 1 & 5 & 6 \\
Total & 100 & 9 & 23 & 32 \\
\hline
\end{tabular}

(D1/week 4 and D2/week 4) values for each diet. Leastsquares means and CI were calculated for the differences among D1/week 0, D2/week 0, D1/week 4 and D2/week 4, as well as for the net difference between the diets, i.e. (D1/week 4-D1/week 0)-(D2/week 4-D2/week 0). Another set of analyses with body weight included as a continuous covariate was carried out. The analyses were carried out using SAS PROC Mixed (v. 8.2, SAS Institute, Inc.). Using the Framingham Study equation ${ }^{(22)}$, 10-year CHD risk was calculated by considering age, sex, total cholesterol and HDL-cholesterol levels, smoking and systolic BP values. Blood marker and anthropometric data, including BP, were also subjected to a multivariate analysis (orthogonal partial least-squares discriminant analysis) using the SIMCA 13.0 software (MKS Umetrics $\mathrm{AB})^{(23)}$ for estimating overall difference between D1 and D2. Pearson's correlation analyses, using the MINITAB Statistical Software (release 13.32; Minitab, Inc.), were used to study relationships among the different biomarkers, satiety scores and results from the breath hydrogen tests. Values of $P \leq 0.05$ were considered statistically significant.

\section{Power calculation}

The primary outcome measure was change in plasma LDLcholesterol levels. Assuming a $0.35 \mathrm{mmol} / 1$ (approximately $10 \%)$ post-diet difference and a $0.8 \mathrm{sD}^{(24)}$, with $\alpha=0.05$ and $1-\beta=0 \cdot 8$, a minimum of thirty-eight participants were required.

\section{Results}

\section{Study population and dietary compliance}

Baseline data (Table 1) confirmed the overall healthy condition of the cohort studied. In terms of blood lipids, the group can be considered to be mildly hypercholesterolaemic, borderline high for LDL-cholesterol, but with HDL-cholesterol and TAG values within the desirable range.

Adherence to both the dietary plans was good, with slightly greater compliance for D2 than for D1 (99 (SEM 0.5) v. 97 (SEM $0.3) \%$, respectively; $P=0.003)$. D1 was described as more satiating than the habitual diet by $75 \%$ of the participants, while $63 \%$ reported this sensation for D2. The overall daily satiety feeling with D1 was significantly greater than that with D2 $(P=0 \cdot 001)$. Increased intestinal gas production was experienced by forty-one participants $(90 \%)$ with D1 than with their regular regimens. However, the majority of them (thirty-six subjects, i.e. $88 \%$ ) did not find this as a burden.

\section{Anthropometric variables}

In accordance with the study design, body weight and BMI values did not change with any of the diets. No variation in waist circumference could be recorded either (Table 5).

\section{Blood variables}

Compared with baseline values, both diets reduced total cholesterol, LDL-cholesterol and HDL-cholesterol levels (Table 5). However, D1 had more pronounced effects on total cholesterol and LDL-cholesterol levels than D2 $(P=0.01$ and $P=0.0029$, respectively). Similarly, D1 had greater effect on apo fractions, particularly on apoB. None of the diets altered the LDL:HDL or apoB:apoA1 ratios.

Neither the glycaemic control variables measured (fasting glucose, insulin, homeostatic model assessment of insulin resistance and glycated $\mathrm{Hb}$ ) nor the inflammatory marker C-reactive protein was affected by the diets. A significant decrease in circulating GGT levels was observed after D1 consumption $(P=0.05)$, but not after D2 consumption.

Table 4. Representative $1 \mathrm{~d}$ menus for diet 1 (D1, functional diet) and diet 2 (D2, control diet)

\begin{tabular}{|c|c|}
\hline D1 & $\mathrm{D} 2$ \\
\hline \multicolumn{2}{|l|}{ Breakfast } \\
\hline Milk (1.5\% fat) & Wheat-bran cereal \\
\hline Whole-kernel barley porridge & Sour milk ( $1.5 \%$ fat $)$ \\
\hline Dried apricots & Apple purée \\
\hline Apple purée & Wheat bread \\
\hline Whole-kernel barley bread & Margarine (40\% fat) \\
\hline Lean ham (maximum 3\% fat) & Liver pâté (25\% fat) \\
\hline Bell pepper & Cucumber \\
\hline Orange & Orange \\
\hline \multicolumn{2}{|l|}{ Lunch } \\
\hline Whole-kernel barley bread & Smoked pork minestrone \\
\hline Cod gratin with chickpeas & Apple pie with custard \\
\hline Rye crisp bread & Margarine (butter-oil blend) \\
\hline Salad & \\
\hline \multicolumn{2}{|l|}{ Dinner } \\
\hline Whole-kernel barley bread & Wheat bread \\
\hline Cheese (28\% fat) & Margarine ( $40 \%$ fat) \\
\hline Tomatoes & Cheese (28\% fat) \\
\hline Camembert cheese (23\% fat) & Bell pepper \\
\hline Lettuce & Liver pâté ( $25 \%$ fat) \\
\hline \multirow[t]{4}{*}{ Pesto sauce } & Pickled cucumber \\
\hline & Cottage cheese \\
\hline & Creamed salmon spread \\
\hline & $\begin{array}{l}\text { Mixed fruits (at least three } \\
\text { per d) }\end{array}$ \\
\hline \multicolumn{2}{|l|}{ Snack } \\
\hline Fruit, two portions per d & Fruit, two portions per $\mathrm{d}$ \\
\hline Whole-kernel barley bread & Margarine (40\% fat) \\
\hline \multirow[t]{4}{*}{ Roasted brown beans } & Wheat bread \\
\hline & Wheat crisp rolls \\
\hline & Marmalade \\
\hline & Cinnamon roll \\
\hline
\end{tabular}


Table 5. Effect of diet 1 (D1) and diet 2 (D2) on anthropometry, blood parameters, blood pressure, exhaled hydrogen and estimated cardiovascular risk (Mean values with their standard errors, $n 46$ )

\begin{tabular}{|c|c|c|c|c|c|c|c|c|c|c|c|c|}
\hline & \multicolumn{5}{|c|}{ D1 (barley + legumes) } & \multicolumn{5}{|c|}{ D2 (control) } & \multirow[b]{3}{*}{$P(\mathrm{D} 1-\mathrm{D} 2) \dagger$} & \multirow{3}{*}{$\begin{array}{c}P(\mathrm{D} 1-\mathrm{D} 2) \ddagger \\
\text { weight adjustec }\end{array}$} \\
\hline & \multicolumn{2}{|c|}{ Week 0} & \multicolumn{2}{|c|}{ Week 4} & \multirow[b]{2}{*}{ Change (\%) } & \multicolumn{2}{|c|}{ Week 0} & \multicolumn{2}{|c|}{ Week 4} & \multirow[b]{2}{*}{ Change (\%) } & & \\
\hline & Mean & SEM & Mean & SEM & & Mean & SEM & Mean & SEM & & & \\
\hline Body weight (kg) & 77.9 & 1.3 & 77.4 & 1.3 & -0.6 & $78 \cdot 1$ & 1.4 & 77.5 & $1 \cdot 3$ & -0.7 & 0.8120 & \\
\hline BMI $\left(\mathrm{kg} / \mathrm{m}^{2}\right)$ & $28 \cdot 7$ & 0.3 & 28.5 & 0.3 & -0.6 & $28 \cdot 7$ & 0.3 & 28.5 & 0.3 & -0.7 & 0.8120 & \\
\hline Waist circumference $(\mathrm{cm})$ & $96 \cdot 6$ & $1 \cdot 1$ & $97 \cdot 1$ & $1 \cdot 1$ & 0.5 & $97 \cdot 6$ & $1 \cdot 1$ & $96 \cdot 8$ & $1 \cdot 1$ & -0.8 & 0.2005 & 0.1545 \\
\hline \multicolumn{13}{|l|}{ Cholesterol (mmol/l) } \\
\hline Total§ & $6 \cdot 37$ & $0 \cdot 16$ & $5 \cdot 62^{\star \star \star}$ & $0 \cdot 16$ & -12 & $6 \cdot 42$ & 0.17 & $5 \cdot 97^{\star \star}$ & $0 \cdot 17$ & -7 & 0.0101 & 0.0106 \\
\hline LDL§ & 4.03 & 0.15 & $3.59^{\star \star \star}$ & 0.13 & -11 & 4.01 & $0 \cdot 16$ & $3 \cdot 84^{\star \star}$ & 0.14 & -3 & 0.0029 & 0.0030 \\
\hline HDL§ & 1.82 & 0.08 & $1.61^{\star \star \star}$ & 0.07 & -11 & 1.84 & 0.08 & $1 \cdot 66^{\star \star \star}$ & 0.07 & -8 & 0.5208 & 0.5309 \\
\hline TAG $(\mathrm{mmol} / \mathrm{l})$ & $1 \cdot 15$ & 0.09 & 1.08 & 0.06 & -4 & $1 \cdot 10$ & 0.08 & $1 \cdot 12$ & 0.07 & 4 & 0.2119 & 0.2061 \\
\hline \multicolumn{13}{|l|}{ Apo $(g / l)$} \\
\hline ApoA1§ & 1.74 & 0.04 & $1.53^{\star \star \star}$ & 0.04 & -11 & 1.73 & 0.04 & $1.59^{\star \star \star}$ & 0.04 & -8 & 0.0990 & 0.1003 \\
\hline ApoB\| & $1 \cdot 12$ & 0.04 & $1 \cdot 02^{\star *}$ & 0.04 & -8 & $1 \cdot 12$ & 0.05 & 1.09 & 0.04 & -2 & 0.0024 & 0.0024 \\
\hline \multicolumn{13}{|l|}{ Ratios } \\
\hline LDL:HDL & 2.46 & $0 \cdot 16$ & 2.46 & 0.17 & 1 & $2 \cdot 40$ & 0.16 & 2.50 & 0.15 & 4 & 0.2429 & 0.2414 \\
\hline ApoB:apoA1 & 0.67 & 0.03 & 0.69 & 0.03 & 5 & 0.67 & 0.04 & 0.71 & 0.03 & 6 & 0.5353 & 0.5317 \\
\hline Insulin (mU/l)ף & 7.44 & 0.50 & $7 \cdot 29$ & 0.39 & -1 & $7 \cdot 13$ & 0.42 & 7.35 & 0.45 & 9 & 0.4982 & 0.5058 \\
\hline Glucose $(\mathrm{mmol} / \mathrm{l})$ & 5.33 & 0.06 & 5.45 & 0.67 & 2 & 5.41 & 0.08 & 5.38 & 0.07 & -1 & 0.1336 & 0.1361 \\
\hline HOMA-IR & 1.60 & $0 \cdot 12$ & 1.60 & 0.09 & 0 & 1.57 & 0.11 & 1.61 & 0.11 & 2 & 0.7785 & 0.7909 \\
\hline HbA1c (\%) & 3.67 & 0.05 & 3.72 & 0.05 & 1 & 3.65 & 0.04 & $3 \cdot 70$ & 0.05 & 2 & 0.7855 & 0.7734 \\
\hline GGT $(\mu \mathrm{kat} / \mathrm{l}) \|$ & 0.37 & 0.03 & $0.33^{*}$ & 0.03 & -8 & 0.33 & 0.03 & 0.34 & 0.03 & 3 & 0.0228 & 0.0133 \\
\hline $\operatorname{CRP}(\mathrm{mg} / \mathrm{l})$ & 2.03 & 0.22 & 1.83 & 0.26 & -5 & 1.89 & 0.22 & 1.86 & 0.34 & -2 & 0.4792 & 0.4795 \\
\hline \multicolumn{13}{|l|}{ Blood pressure $(\mathrm{mmHg})$} \\
\hline Systolic & 134 & 3 & 131 & 2 & -2 & 133 & 3 & 133 & 3 & 0 & 0.2232 & 0.2308 \\
\hline Diastolic§ & 84 & 1 & $81^{*}$ & 1 & -3 & 82 & 1 & 82 & 1 & 0 & 0.0178 & 0.0183 \\
\hline Breath hydrogen $(\mathrm{ppm}) \S$ & 9.9 & $1 \cdot 2$ & $23 \cdot 3^{\star *}$ & $3 \cdot 2$ & 135 & $9 \cdot 6$ & 1.4 & $12 \cdot 9$ & $1 \cdot 3$ & 34 & 0.0071 & 0.0072 \\
\hline Framingham 10-year risk\| & $3 \cdot 0$ & 0.3 & $2 \cdot 7^{\star}$ & 0.3 & -11 & 2.9 & 0.2 & $3 \cdot 0$ & 0.3 & 6 & 0.0152 & 0.0157 \\
\hline
\end{tabular}

HOMA-IR, homeostatic model assessment of insulin resistance; HbA1c, glycated Hb; GGT, $\gamma$-glutamyl transferase; CRP, C-reactive protein; ppm, parts per million.

Mean values were significantly different from baseline (week 0): ${ }^{*} P<0.05$; ${ }^{\star *} P<0.001$; $* \star * * 0.0001$.

$\uparrow$ value for the comparison of changes after active diet and control diet consumption.
$\ddagger P$ value for the comparison of changes after active diet and control diet consumption, after adjustment for weight change.

$\S$ The overall effect of diet was significant $(P<0.0001)$.

$\|$ The overall effect of diet was significant $(P<0.05)$.

ๆ $1 \mathrm{mU} / \mathrm{l}=6.945 \mathrm{pmol} / \mathrm{l}$. 


\section{Blood pressure and cardiovascular risk}

A significantly reduced $(-3 \%, P<0.05)$ diastolic BP value was observed after D1 consumption, whereas D2 had no effect. None of the diets affected systolic BP. An 11\% lower $(P<0.05)$ overall cardiovascular risk score was calculated after D1 consumption, with no significant effect being observed after D2 consumption (Table 5).

\section{Breath hydrogen}

D1 promoted a $2 \cdot 3$-fold increase in fasting exhaled hydrogen levels $(P<0.001)$, while D2 had no significant effect (Table 5). The variation in breath hydrogen levels after D1 consumption exhibited a negative correlation with the change in systolic BP $(r-0.30$ and $P=0.045)$. No other significant correlation among the different biomarkers could be recorded.

\section{Discussion}

A diet enriched with whole-grain kernel-based barley products, brown beans and chickpeas (D1) promoted reductions in plasma total cholesterol, LDL-cholesterol and apoliprotein B levels beyond those observed with a reference regimen rich in dietary fibre and complying with sound dietary recommendations. In addition to these changes in blood lipid profile, D1 decreased two other parameters associated with cardiometabolic risk, i.e. circulating GGT and BP. Furthermore, D1 also resulted in an $11 \%$ drop in the Framingham cardiovascular risk estimate. Since the study was carried out in a cohort of mature women who had no medical condition besides mild hypercholesterolaemia, the present results may be of value for developing dietary strategies for the prevention of cardiometabolic diseases.

Overall, D1 promoted a $50 \%$ greater improvement of risk markers than D2 $(P<0 \cdot 0001)$, as estimated by the multivariate data analysis. The limited, but yet significant, effect of the reference diet (D2) on blood lipid profile reflects the improved nutritional quality of this regimen, including better fat composition and substantially increased dietary fibre supply (Table 2) in comparison with the habitual diet of the participants.

The important reduction in blood cholesterol fractions induced by $\mathrm{D} 1$ can be explained by the combined action of the functional components present in the two legumes and barley grains included in the diet. Whole barley kernels ${ }^{(12,25)}$ common beans $^{(26)}$ and chickpeas ${ }^{(27)}$ have recognised lowglycaemic index features, and it has been shown that low-GI diets promote significantly improved lipid profiles in medium- and longer-term interventions, particularly with regard to lowered total cholesterol and LDL-cholesterol levels ${ }^{(28-30)}$

Dietary fibre quality is another factor that may have played a role in the observed blood lipid response to D1. Diets having significant amounts of viscous fibres, such as $\beta$-glucans in barley $^{(31)}$ and the soluble indigestible fractions that characterise legume seeds ${ }^{(32)}$, are acknowledged as cholesterol lowering ${ }^{(32-34)}$. Besides the physico-chemical effects that viscous fibres have on the digestive/absorptive phase in the small intestine, the colonic fermentation of carbohydrates escaping ileal digestion may also have a beneficial impact on cholesterolaemia, via SCFA production. Propionate, in particular, acts as a negative modulator of hepatic cholesterol synthesis ${ }^{(5,34)}$. The indigestible fraction of cooked legumes and intact barley kernels is susceptible to fermentation by gut bacteria. In fact, dietary fibre, resistant starch and $\alpha$-galactosides in legumes ${ }^{(35-38)}$, as well as $\beta$-glucans in barley ${ }^{(39)}$, have been suggested as prebiotics. This idea is supported by recent evidence from meal studies carried out for a duration of $10-16 \mathrm{~h}$ indicating the prebiotic effects of the intrinsic indigestible fraction present in these foods ${ }^{(13,16)}$. Moreover, supplementation of white wheat bread with resistant starch and dietary fibre from barley in amounts mimicking those of these carbohydrates present in barley kernel products has been shown to improve glucose regulation in healthy subjects, presumably through a mechanism involving gut fermentation $^{(14)}$. Thus, the potential prebiotic action of legumes and barley has been proposed as a link between the consumption of these foods and the decreased levels of circulating markers of inflammation, with perceived impacts on cardiometabolic risk $^{(14,16)}$. Polyphenols and phenolic acids, which are also abundant in common beans, chickpeas and whole barley kernels ${ }^{(40,41)}$, may be additional substrates for colonic fermentation $^{(42)}$. The markedly higher breath hydrogen levels associated with D1 speak in favour of a mechanism associated with prebiotic effects. In fact, changes in exhaled hydrogen levels after D1 consumption exhibited a negative correlation with the change in systolic BP $(r-0.30$ and $P=0.045)$.

Circulating GGT is another interesting biomarker the levels of which decreased with D1 consumption. High levels of this enzyme are indicative of non-alcoholic liver disease and are associated with incident diabetes, hypertension and vascular events $^{(43)}$. Recently, GGT activity has also been proposed as an independent predictor of the incidence of cardiometabolic risk $^{(44,45)}$. Among the possible mechanism(s) behind the positive association between GGT and cardiometabolic risk, inflammation-related mechanisms may be involved ${ }^{(45)}$. Only a few studies have examined the influence of diet functionality on $\mathrm{GGT}^{(46,47)}$. Further investigation on the dietary modulation of this emerging risk marker may give deeper insights into its usefulness in preventive nutrition.

In spite of its relative simplicity, D1 has important antiinflammatory, cholesterol-lowering, low-glycaemic index and potential prebiotic features, which are substantial components of effective multifunctional metabolic protective diets ${ }^{(6)}$. This fact may be advantageous for sustaining adequate adherence to treatment in prolonged experimental trials or under common living conditions.

In full compliance with one of the study design premises, the beneficial action of D1 was observed in the absence of significant body weight reduction. Considering the appreciable satiating power mentioned by most participants, it is tempting to speculate about possible additional metabolic improvement under conditions where reduced voluntary intake may lead to weight loss. Such a possibility, as well as the potential impact 
of D1 on food intake-regulating hormones, may require further investigation.

Due to practical reasons, the present study was carried out in an exclusively female cohort, which limits the extrapolation of the results to the general population. Nonetheless, the general amelioration recorded is worth noting, since the association between cardiovascular events and altered metabolic profiles is greater in women than in men ${ }^{(48,49)}$ and, in addition, responses to pharmacological treatments for dyslipidaemia are more limited in women than in males ${ }^{(50)}$.

\section{Conclusion}

A diet combining the functional features of legumes and kernel-based barley products reduced the levels of different cardiometabolic diseases-related markers, including a comprehensive cardiovascular risk estimate, in a group of mature overweight women. The results are in accordance with previous evidence for the potential prebiotic beneficial action of these foods. This type of diet has potential for the dietary prevention of cardiometabolic diseases.

\section{Acknowledgements}

The authors thank Dr Peter Höglund (Region Skåne Competence Centre for Clinical Research, Lund) and Dr Olof Rosén (MKS Umetrics AB, Malmö) for their assistance with the statistical analysis. They also thank Ulla Johansson's for her skilful support in designing the menus and providing dietary coaching as well as Ingrid Ristilammi's for her help in recruiting and logistics management of participants.

The present study was funded by the Lund University Antidiabetic Food Centre, a VINNOVA VINN Excellence Centre, which had no role in the design and analysis of the study or in the writing of this article.

The authors' contributions are as follows: I. B., J. T., A. N. and M. J. designed the research; A. N. and J. T. coordinated the dietetic instruction and coaching of the subjects; J. T. conducted the research and coordinated the whole study; J. T. and A. N. analysed the data; J. T. drafted the manuscript; J. T. and I. B. had primary responsibility for the final content. All authors revised, discussed and approved the final paper.

None of the authors has any conflicts of interest.

\section{References}

1. Feldeisen SE \& Tucker KL (2007) Nutritional strategies in the prevention and treatment of metabolic syndrome. Appl Physiol Nutr Metab 32, 42-60.

2. Bruckert E \& Rosenbaum D (2011) Lowering LDL-cholesterol through diet: potential role in the statin era. Curr Opin Lipidol 22, 43-48.

3. Adamsson V, Reumark A, Fredriksson IB, et al. (2011) Effects of a healthy Nordic diet on cardiovascular risk factors in hypercholesterolaemic subjects: a randomized controlled trial (NORDIET). J Intern Med 269, 150-159.

4. Estruch R, Martínez-González MA, Corella D, et al. (2006) Effects of a Mediterranean-style diet on cardiovascular risk factors: a randomized trial. Ann Intern Med 145, 1-11.
5. Jenkins DJA, Kendall CWC, Faulkner D, et al. (2002) A dietary portfolio approach to cholesterol reduction: combined effects of plant sterols, vegetable proteins, and viscous fibers in hypercholesterolemia. Metabolism 51, 1596-1604.

6. Tovar J, Nilsson A, Johansson M, et al. (2012) A diet based on multiple functional concepts improves cardiometabolic risk parameters in healthy subjects. Nutr Metab (Lond) 9, 29.

7. Jenkins DJ, Kendall CW, Faulkner DA, et al. (2006) Assessment of the longer-term effects of a dietary portfolio of cholesterol-lowering foods in hypercholesterolemia. Am J Clin Nutr 83, 582-591.

8. Venn BJ, Perry T, Green TJ, et al. (2010) The effect of increasing consumption of pulses and whole grains in obese people: a randomized controlled trial. J Am Coll Nutr 29, 365-372.

9. Hutchins AM, Winham DM \& Thompson SV (2012) Phaseolus beans: impact ion glycaemic response and chronic disease risk in human subjects. BrJ Nutr 108, Suppl. 1, S52-S65.

10. Behall KM, Scholfield DJ \& Hallfrisch J (2006) Whole-grain diets reduce blood pressure in mildly hypercholesterolemic men and women. J Am Diet Assoc 106, 1445-1449.

11. Björck I, Ostman E, Kristensen M, et al. (2012) Cereal grains for nutrition and health benefits: overview of results from in vitro, animal and human studies in the HEALTHGRAIN project. Trends Food Sci Technol 25, 87-100.

12. Nilsson A, Granfeldt Y, Östman E, et al. (2006) Effects of GI and content of indigestible carbohydrates of cereal-based evening meals on glucose tolerance at a subsequent standardized breakfast. Eur J Clin Nutr 60, 1092-1099.

13. Nilsson A, Östman E, Preston T, et al. (2008) Effects of GI vs content of cereal fibre of the evening meal on glucose tolerance at a subsequent standardized breakfast. Eur J Clin Nutr 62, $712-720$.

14. Nilsson AC, Ostman EM, Holst JJ, et al. (2008) Including indigestible carbohydrates in the evening meal of healthy subjects improves glucose tolerance, lowers inflammatory markers, and increases satiety after a subsequent standardized breakfast. J Nutr 138, 732-739.

15. Tovar J (1994) Bioavailability of carbohydrates in legumes. Digestible and indigestible fractions. Arch Latinoamer Nutr 44, 36S-40S.

16. Nilsson A, Johansson E, Ekström L, et al. (2013) Effects of a brown beans evening meal on metabolic risk markers and appetite regulating hormones at a subsequent standardized breakfast: a randomized cross-over study. PLOS ONE 8, e59985.

17. Nordic Council of Ministers (2004) Nutrition Recommendations 2004: Integrating Nutrition and Physical Activity, 4th ed. Copenhagen: Nordic Council of Ministers.

18. Friedewald WT, Levy RI \& Fredriksson DS (1972) Estimation of the concentration of low-density lipoprotein cholesterol in plasma, without use of the preparative ultracentrifuge. Clin Chem 18, 499-502.

19. Matthews DR, Hosker JP, Rudenski AS, et al. (1985) Homeostasis model assessment: insulin resistance and beta-cell function from fasting plasma glucose and insulin concentrations in man. Diabetologia 28, 412-419.

20. Asp NG, Johansson CG, Hallmer H, et al. (1983) Rapid enzymatic assay of insoluble and soluble dietary fibre. J Agric Food Chem 31, 476-482.

21. Åkerberg AKE, Liljeberg HGM \& Granfeldt Y (1998) An in vitro method, based on chewing, to predict resistant starch content in foods allows parallel determination of potentially available starch and dietary fibre. J Nutr 128, 651-659.

22. Anderson KM, Wilson PWF, Odell PM, et al. (1991) An updated coronary risk profile - a statement for healthprofessionals. Circulation 83, 356-362. 
23. Eriksson L, Johansson E, Kettaneh-Wold N, et al. (2006) Multi- and Megavariate Data Analysis, Part II, Advanced Applications and Method Extensions, Chapter 23. Umeå: Umetrics Academy.

24. Daryani A, Berglund L, Andersson Å, et al. (2005) Risk factors for coronary heart disease among immigrant women from Iran and Turkey, compared to women of Swedish ethnicity. Ethn Dis 15, 213-220.

25. Foster-Powell K, Holt SHA \& Brand-Miller JC (2002) International table of glycemic index and glycemic load values: 2002. Am J Clin Nutr 76, 5-56.

26. Tovar J, Granfeldt Y \& Björck I (1992) Effect of processing on blood-glucose response to starch in legumes. J Agric Food Chem 40, 1846-1851.

27. Nestel P, Cehun M \& Chronopoulus A (2004) Effects of longterm consumption and single meals of chickpeas on plasma glucose, insulin and triacylglycerol concentrations. Am J Clin Nutr 79, 390-395.

28. Frost G, Leeds AA, Dore CJ, et al. (1999) Glycaemic index as a determinant of serum HDL-cholesterol concentration. Lancet 353, 1045-1048.

29. Sloth B, Krog-Mikkelsen I, Flint A, et al. (2004) No difference in body weight decrease between a low-glycemic-index and a high-glycemic-index diet but reduced LDL cholesterol after 10-wk ad libitum intake of the low-glycemic-index diet. $A m$ J Clin Nutr 80, 337-347.

30. de Rougemont A, Normand S, Nazare JA, et al. (2007) Beneficial effects of a 5-week low-glycaemic index regimen on weight control and cardiovascular risk factors in overweight non-diabetic subjects. Br J Nutr 98, 1288-1298.

31. Biörklund M, van Rees A, Mensink RP, et al. (2005) Changes in serum lipids and postprandial glucose and insulin concentrations after consumption of beverages with glucans from oats or barley: a randomised dose-controlled trial. Eur J Clin Nutr 59, 1272-1281.

32. Viuda-Martos M, Lopez-Marcos MC, Fernandez-Lopez J, et al (2010) Role of fiber in cardiovascular diseases: a review. Compr Rev Food Sci Food Saf 9, 240-258.

33. Jenkins DJA, Kendall CWC, Vuksan V, et al. (2002) Soluble fiber intake at a dose approved by the US Food and Drug Administration for a claim of health benefits: serum lipid risk factors for cardiovascular disease assessed in a randomized controlled crossover trial. Am J Clin Nutr 75, 834-839.

34. Finley JW, Burrell JB \& Reeves PG (2007) Pinto bean consumption changes SCFA profiles in fecal fermentations, bacterial populations of the lower bowel, and lipid profiles in blood of humans. J Nutr 137, 2391-2398.

35. Tovar J, Björck I \& Asp N-G (1992) Incomplete digestion of legume starches in rats: a study of precooked flours containing retrograded and physically inaccessible starch fractions. J Nutr 122, 1500-1507.

36. Queiroz-Monici SK, Costa EAG, Silva N, et al. (2005) Bifidogenic effect of dietary fiber and resistant starch from leguminous on the intestinal microbiota of rats. Nutrition 21, 602-608.
37. Martinez-Villaluenga C, Frias J \& Vidal-Valverde C (2008) Alpha-galactosides: antinutritional factors or functional ingredients? Crit Rev Food Sci Nutr 48, 301-316.

38. Maathuis AJ, van den Heuvel EG, Schoterman $\mathrm{MH}$, et al. (2012) Galacto-oligosaccharides have prebiotic activity in a dynamic in vitro colon model using a C-labeling technique. J Nutr 142, 1205-1212.

39. Mitsou EK, Panopoulou N, Turunen K, et al. (2010) Prebiotic potential of barley derived beta-glucan at low intake levels: a randomised, double-blinded, placebo-controlled clinical study. Food Res Int 43, 1086-1092.

40. Hernandez-Salazar M, Osorio-Diaz P, Loarca-Pina G, et al. (2010) In vitro fermentability and antioxidant capacity of the indigestible fraction of cooked blackbeans (Phaseolus vulgaris L.), lentils (Lens culinaris L.) and chickpeas (Cicer arietinum L.). J Sci Food Agric 90, 1417-1422.

41. Gamel T \& Abdel-Aal EM (2012) Phenolic acids and antioxidant properties of barley wholegrain and pearling fractions. Agric Food Sci 21, 118-131.

42. Parkar SG, Stevenson DE \& Skinner MA (2008) The potential influence of fruit polyphenols on colonic microflora and human gut health. Int J Food Microbiol 124, 295-298.

43. Fraser A, Harris R, Sattar N, et al. (2007) Gamma-glutamyltransferase is associated with incident vascular events independently of alcohol intake analysis of the British women's heart and health study and meta-analysis. Arterioescler Thromb Vasc Biol 27, 2729-2735.

44. Montonen J, Drogan D, Joost H-G, et al. (2011) Estimation of the contribution of biomarkers of different metabolic pathways to risk of type 2 diabetes. Eur J Epidemiol 26, 29-38.

45. Liu C-F, Zhou W-N \& Fang N-Y (2012) Gamma-glutamyltransferase levels and risk of metabolic syndrome: a meta-analysis of prospective cohort studies. Clin Pract $\mathbf{6 6}$, 692-698.

46. Aller R, De Luis DA, Izaola O, et al. (2011) Effect of a probiotic on liver aminotransferases in nonalcoholic fatty liver patients: a double blind randomized clinical trial. Eur Rev Med Pharmacol Sci 15, 1090-1095.

47. Franzini L, Ardigò D, Valtuena S, et al. (2012) Food selection based on high antioxidant capacity improves endothelial function in a low cardiovascular risk population. Nutr Metab Cardiovasc Dis 22, 50-57.

48. Gami AS, Witt BJ, Howard DE, et al. (2007) Metabolic syndrome and risk of incident cardiovascular events and death: a systematic review and meta-analysis of longitudinal studies. J Am Coll Cardiol 49, 403-414.

49. Rachas A, Raffaitin C, Barberger-Gateau P, et al. (2012) Clinical usefulness of the metabolic syndrome for the risk of coronary heart disease does not exceed the sum of its individual components in older men and women. The Three-City (3C) Study. Heart 98, 650-655.

50. Mehner A, Lindblad U, Råstam L, et al. (2008) Cholesterol in women at high cardiovascular risk is less successfully treated than in corresponding men. The Skaraborg Hypertension and Diabetes Project. Eur J Clin Pharmacol 64, 815-820. 